\title{
Armazenamento de carbono em frações lábeis da matéria orgânica de um Latossolo Vermelho sob plantio direto
}

\author{
Cimélio Bayer(1), Ladislau Martin-Neto ${ }^{(2)}$, João Mielniczuk ${ }^{(1)}$ e Aurélio Pavinato(3)
}

\begin{abstract}
(1)Universidade Federal do Rio Grande do Sul, Dep. de Solos, Caixa Postal 15100, CEP 90001-970 Porto Alegre, RS. E-mail: cimelio.bayer@ufrgs.br, mieln@vortex.ufrgs.br (2)Embrapa Instrumentação Agropecuária, Caixa Postal 741, CEP 13560-970 São Carlos, SP. E-mail: martin@cnpdia.embrapa.br (3) SLC Agrícola, Caixa Postal 13200, CEP 98920-000 Porto Alegre, RS. E-mail: pavinato@slcagricola.com.br
\end{abstract}

\begin{abstract}
Resumo - A reserva de carbono na matéria orgânica (MO) do solo é uma importante estratégia para atenuar a concentração de dióxido de carbono na atmosfera. O objetivo deste trabalho foi avaliar o efeito do plantio direto (PD), durante seis anos, sob quatro sistemas de cultura de outono na sucessão comercial soja-milho (guanduanão-milheto, crotalária-sorgo, girassol-aveia-preta e nabo forrageiro-milho), no armazenamento de carbono nas frações particulada $(>53 \mu \mathrm{m})$ e associada aos minerais $(<53 \mu \mathrm{m})$ da MO de um Latossolo Vermelho argiloso da Região do Cerrado. Em comparação ao preparo convencional (PC) com cultivo de culturas estivais, o PD aumentou os estoques de carbono orgânico total (COT) na camada superficial do solo $(0-5 \mathrm{~cm})$. Na camada de $0-20 \mathrm{~cm}$, o solo sob nabo forrageiro-milho manteve um estoque de COT superior em $9 \%\left(4,66 \mathrm{Mg} \mathrm{ha}^{-1}\right)$ ao do solo em PC $\left(54,30 \mathrm{Mg} \mathrm{ha}^{-1}\right)$. Nos demais sistemas de culturas, o estoque de COT nessa camada não diferiu do solo em PC. $\mathrm{O}$ estoque de $\mathrm{C}$ na $\mathrm{MO}$ particulada foi mais sensível ao manejo do solo do que o COT, com um incremento entre $37 \%$ e $52 \%$ sob PD, em comparação ao PC (0-20 cm). O estoque de C na $\mathrm{MO}$ associada aos minerais não foi afetado pelos sistemas de manejo, o que pode estar relacionado ao curto período sob $\mathrm{PD}$ e à microagregação altamente estável. $\mathrm{O}$ armazenamento de $\mathrm{C}$ preferencialmente na fração lábil da MO do solo representa um benefício ambiental cuja manutenção depende fundamentalmente da continuidade do plantio direto e do contínuo aporte de resíduos vegetais.
\end{abstract}

Termos para indexação: solos tropicais, efeito estufa, agricultura conservacionista, manejo do solo.

\section{Carbon storage in labile fractions of soil organic matter in a tropical no-tillage Oxisol}

\begin{abstract}
Carbon storage in the soil organic matter (SOM) is an important strategy to mitigate carbon dioxide concentration in the atmosphere. The objective of this study was to evaluate the effect of the no-tillage (NT) use for six years, under four autumn (dry-season) cover crops based maize and soybean crop rotations (pigeon peapearl millet, bengan hemp-sorghum, sunflower-black oat, and wild radish-maize), on C storage in the particulate $(>53 \mu \mathrm{m})$ and mineral-associated $(<53 \mu \mathrm{m})$ SOM fractions of a clayey Oxisol from Brazilian Cerrado region. In comparison to conventional tillage (CT) under summer cash-crops, NT increased the total organic carbon (TOC) stocks in the surface soil layer $(0-5 \mathrm{~cm})$. In the top $20 \mathrm{~cm}$ layer, NT soil under wild radish-maize had $9 \%$ (4.66 $\mathrm{Mg} \mathrm{ha}^{-1}$ ) more TOC than the conventionally tilled soil. On the other hand, C storage in NT soil under other cropping systems was statiscally similar to the $\mathrm{CT}$ soil. The $\mathrm{C}$ stocks in the particulate SOM fraction increased by 37 to $52 \%$ in NT soil $(0-20 \mathrm{~cm})$ in comparison to $\mathrm{CT}$. The higher sensitivity to soil management changes made the particulate organic matter a more adequate soil quality index compared to the TOC stocks. The $\mathrm{C}$ stock in the mineral-associated SOM fraction was not affected by soil management systems, which can be related to the short-term under NT and or to the highly stable soil microaggregates in this clayey Oxisol. The preferential C storage in the labile SOM fraction is an environmental benefit, which is expected to occur only under continuous no-tillage and crop residues addition.
\end{abstract}

Index terms: tropical soils, greenhouse effect, conservative agriculture, soil management.

\section{Introdução}

A Região do Cerrado brasileiro abrange 207 milhões de hectares, representando aproximadamente $4 \%$ da região tropical mundial. Atualmente, uma área superior a 12 milhões de hectares dessa região é cultivada com culturas anuais, e são utilizados diversos sistemas de preparo do solo, com predomínio de uso de grade pesada e de arado de discos, além de arado de aivecas e 
escarificador. O manejo do solo com sistemas de revolvimento intenso para cultivo de culturas anuais tem acelerado o processo de degradação das condições químicas, físicas e biológicas dos solos nesta região (Resck, 1997).

O estoque de matéria orgânica (MO) apresenta rápida queda quando o solo é submetido a sistemas de preparo com intenso revolvimento (Silva et al., 1994), decorrente do aumento das perdas por erosão hídrica e oxidação microbiana. Esta perda de MO reflete-se negativamente na capacidade de troca de cátions (Silva et al., 1994) e na complexação de elementos tóxicos como o alumínio (Mendonça, 1995) nos solos da região. As boas propriedades físicas que o solo apresenta sob condições naturais são parcialmente perdidas, como a macroagregação, porosidade e infiltração de água (Resck, 1997).

Com a finalidade de reverter o ciclo de degradação do solo e ambiente, a partir da década de 80, iniciou-se a adoção do plantio direto (PD) na região. A evolução das áreas cultivadas em PD foi rápida, e em 2000/2001 aproximadamente 4,9 milhões de hectares já haviam sido cultivados neste sistema de manejo (Associação de Plantio Direto no Cerrado, 2004). A palhada na superfície do solo em PD promove aumento da infiltração e do armazenamento de água no solo, diminuição da temperatura superficial, aumento da atividade microbiana, e acúmulo superficial de nutrientes e de matéria orgânica nas camadas superficiais do solo, entre outros (Bayer \& Mielniczuk, 1999), além de expressiva redução da erosão hídrica (Cassol, 1984).

A acumulação de matéria orgânica em Latossolos da Região do Cerrado sob PD foi verificada por Corazza et al. (1999). Enquanto o solo cultivado com grade pesada apresentou uma perda de $8,3 \mathrm{Mg} \mathrm{ha}^{-1}$ em 12 anos em relação ao solo sob cerrado nativo, no solo em PD verificou-se, em 15 anos, um aumento de $21,4 \mathrm{Mg} \mathrm{ha}^{-1} \mathrm{de}$ $\mathrm{C}$, o que corresponde a uma taxa anual de acúmulo de $\mathrm{C}$ no solo de $1,43 \mathrm{Mg} \mathrm{ha}^{-1}$. Estes resultados demonstram que, enquanto o solo sob $\mathrm{PC}$ atua como uma fonte de $\mathrm{C}$ para atmosfera, o solo em PD passa a atuar como um dreno de $\mathrm{C}$ atmosférico, o que representa uma importante contribuição da agricultura para atenuar a concentração de dióxido de $\mathrm{C}$ na atmosfera e nas alterações climáticas globais.

O C pode acumular em frações lábeis ou estáveis da MO no solo, o que pode ter implicações na durabilidade do seu efeito quanto à retenção de $\mathrm{C}$ atmosférico, bem como nas alterações nas propriedades físicas, químicas e biológicas dos solos sob PD. Assim, visando identifi- car em quais compartimentos o C está acumulando, têm sido utilizadas técnicas de fracionamento físico da MO que podem ser granulométricas (Cambardella \& Elliot, 1992; Feller \& Beare, 1997; Franzluebbers \& Arshad, 1997; Wander et al., 1998; Needelman et al., 1999), densimétricas (Golchin et al., 1994), ou uma mistura de ambas (Six et al., 1998). O acúmulo de C em frações lábeis da MO tem sido relacionado à sua proteção física no interior de agregados, em consequência da inacessibilidade aos microrganismos e suas enzimas (Feller \& Beare, 1997). A MO protegida no interior de agregados apresenta um tempo de permanência no solo maior do que a MO livre, sendo esta proteção maior nos microagregados do que nos macroagregados (Buyanovsky et al., 1994).

O objetivo deste trabalho foi avaliar o efeito da utilização do plantio direto, durante seis anos, sob quatro sistemas de culturas de outono na sucessão comercial soja-milho (guandu-anão-milheto, crotalária-sorgo, girassol-aveia-preta e nabo forrageiro-milho) no armazenamento de carbono nas frações particulada $(>53 \mu \mathrm{m})$ e associada aos minerais $(<53 \mu \mathrm{m})$ da $\mathrm{MO}$ de um Latossolo Vermelho argiloso da Região do Cerrado.

\section{Material e Métodos}

O trabalho foi baseado em amostras de solo coletadas em experimento instalado, em 1993, em área da Fazenda Planalto da Empresa SLC Agrícola, em Costa Rica, MS. O solo é classificado como Latossolo Vermelho ácrico distrófico, com 200, 200 e $600 \mathrm{~g} \mathrm{~kg}^{-1}$ de areia, silte e argila, respectivamente, na camada de 0-20 cm. Segundo análise de difração de raios X, os principais minerais presentes na fração argila são caulinita, óxidos de ferro e gibsita. O clima é classificado como tropical úmido e quente (Aw segundo classificação de Köppen), com uma estação seca de maio a setembro. A temperatura média anual é $24,8^{\circ} \mathrm{C}$, variando mensalmente entre $13,4^{\circ} \mathrm{C}$ (julho) e $31,4^{\circ} \mathrm{C}$ (novembro). A precipitação pluvial anual é de $1.929 \mathrm{~mm}$, e $88 \%$ deste total ocorrem na estação chuvosa. Estas informações foram coletadas na Estação Meteorológica localizada na fazenda, e compreendem valores médios do período de 1992 a 2000.

Previamente à instalação do experimento, o solo foi cultivado durante 10 anos com grade pesada, a uma profundidade aproximada de $17 \mathrm{~cm}$, com as culturas da soja (Glicine max) e milho (Zea mays), sendo este cultivado em apenas três anos. $\mathrm{O}$ experimento consistiu em qua- 
tro sistemas de cultura, dispostos num delineamento em blocos casualizados, com duas repetições. Os sistemas de rotação de cultura consistiram da intercalação anual de culturas outonais (I: guandu-anão (Cajanus cajan)milheto (Pennisetum americanum), II: crotalária (Crotalaria juncea)-sorgo (Sorghum vulgare), III: girassol (Helianthus anuus)-aveia-preta (Avena strigosa), e IV: nabo forrageiro (Raphanus raphanistrum)-milho) com as culturas comerciais soja e milho. Estas combinações de culturas outonais foram dispostas em duas faixas, e, em uma delas, o milho foi cultivado a cada dois anos (50\% da rotação), e na outra, a cada três anos (33\% da rotação). As características químicas do solo (0-20 cm), na média dos sistemas com $33 \%$ e $50 \%$ de milho na rotação, são apresentadas na Tabela 1.

Em todos os anos, as culturas outonais foram semeadas em abril, e a soja e o milho em novembro. O milho e a soja foram semeados com espaçamento de 0,9 e $0,5 \mathrm{~m}$ entre linhas, e a população média foi de aproximadamente $50 \mathrm{mil} \mathrm{e} 220 \mathrm{mil}$ plantas/ha, respectivamente. A semeadura das culturas foi motomecanizada. O manejo das espécies outonais e o controle de ervas daninhas foi realizado com herbicidas com princípio ativo glifosate.

O efeito do PD nos estoques de $\mathrm{C}$ do solo foi avaliado em comparação a um solo em preparo convencional (PC), cultivado com grade pesada, o qual constitui um tratamento de um experimento adjacente ao avaliado neste trabalho, e que apresenta histórico idêntico de manejo do solo ao da área experimental sob PD. Nos seis anos referentes ao período experimental (1993-1998), cultivou-se na área sob PC três safras de soja, duas safras de milho e uma safra de algodão (Hordeum vulgare).

Tabela 1. Características químicas da camada de $0-20 \mathrm{~cm}$ de um Latossolo Vermelho sob preparo convencional e plantio direto em diferentes rotações de cultura.

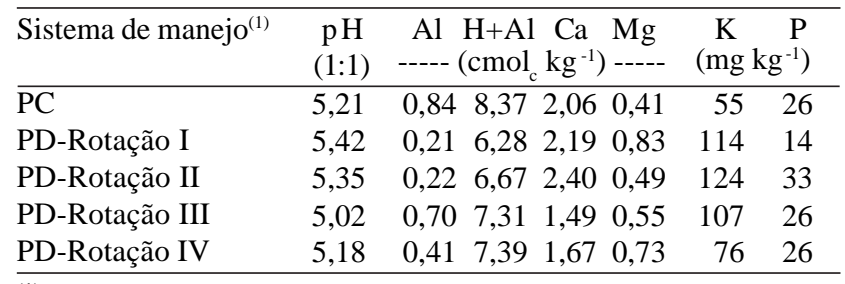

${ }^{(1)} \mathrm{PC}$ : preparo convencional; PD: plantio direto; Rotação I: guanduanão, milheto/soja, milho; Rotação II: crotalária juncea, sorgo/soja, milho; Rotação III: girassol, aveia-preta/soja, milho; Rotação IV: nabo forrageiro, milho/soja, milho.
O solo foi amostrado manualmente em todos os tratamentos, em maio de 1999, nas profundidades de $0-2,5,2,5-5,5-10,10-15$ e 15-20 cm. As amostras de solo foram secadas ao ar, moídas, peneiradas a $2 \mathrm{~mm}$ e analisadas quanto aos teores de C orgânico total (Nelson \& Sommers, 1982). O fracionamento físico da MO foi realizado segundo Cambardella \& Elliot (1992). Aproximadamente $20 \mathrm{~g}$ de solo e $70 \mathrm{~mL}$ de solução de hexametafosfato de sódio $\left(5 \mathrm{~g} \mathrm{~L}^{-1}\right)$ foram agitados durante 15 horas em agitador horizontal. A seguir, a suspensão foi passada em peneira de $53 \mu \mathrm{m}$ com auxílio de jato de água. O material retido na peneira, que consiste na MO particulada, foi secado em estufa a $50^{\circ} \mathrm{C}$, quantificado em relação a sua massa, moído em gral de porcelana e analisado em relação ao teor de $\mathrm{C}$ orgânico. $\mathrm{O}$ estoque de $\mathrm{C}$ na $\mathrm{MO}$ associada aos minerais foi calculado pela diferença entre os estoques de $\mathrm{C}$ na $\mathrm{MO}$ total e na MO particulada $(>53 \mu \mathrm{m})$. A densidade do solo foi determinada mediante coleta de amostras indeformadas em anéis volumétricos metálicos de $5 \times 9 \mathrm{~cm}(\mathrm{hx} \varnothing)$, nas profundidades de $0-5$ e $5-10 \mathrm{~cm}$, e anéis de $10 \times 9 \mathrm{~cm}$, na profundidade de $10-20 \mathrm{~cm}$, sendo os valores de densidade de solo utilizados no cálculo dos estoques de $\mathrm{C}$ no solo.

Os resultados dos estoques de $\mathrm{C}$ no solo foram submetidos à análise da variância. Como não houve diferença entre os resultados com 50\% e 33\% de milho na rotação de culturas, a comparação entre as médias, realizada pelo teste de Tukey a 5\% de probabilidade, foi avaliada na média destes tratamentos, considerando-se, portanto, quatro repetições.

\section{Resultados e Discussão}

O PD promoveu aumento no estoque de COT na camada de 0-20 cm do solo, em comparação ao PC. Entretanto, este efeito foi dependente do sistema de cultura utilizado, e foi significativo somente no tratamento com nabo forrageiro e milho no outono, o qual apresentou aumento médio de $9 \%\left(4,66 \mathrm{Mg} \mathrm{ha}^{-1} \mathrm{de} \mathrm{C}\right)$ no estoque de COT, em comparação ao solo em PC somente com culturas estivais. Nos demais sistemas de cultura, o estoque de COT no solo foi intermediário ao existente nesses dois sistemas (Tabela 2 e Figura 1).

Os maiores aumentos no estoque de COT ocorreram nas camadas superficiais do solo, variando de $39 \%$ a $53 \%$ na camada de $0-2,5 \mathrm{~cm}$ no solo sob PD com culturas outonais e estivais anualmente, em comparação a mesma camada de solo em PC apenas com culturas 
estivais (Tabela 2 e Figura 1). Reicosky et al. (1995) salientam que, mesmo nos casos em que não há aumento de MO na camada arável do solo em PD, o acúmulo de resíduos culturais sobre o solo e de MO nas cama-

Tabela 2. Estoques de C orgânico total (COT) e nas frações particulada (MO particulada) e associada aos minerais (MO associada) da matéria orgânica (MO) de um Latossolo Vermelho sob preparo convencional e plantio direto em diferentes rotações de cultura( ${ }^{(1)}$.

\begin{tabular}{|c|c|c|c|}
\hline \multirow[t]{2}{*}{$\begin{array}{l}\text { Manejo } \\
\text { de solo }\end{array}$} & COT & $\begin{array}{c}\text { MO } \\
\text { particulada }\end{array}$ & $\begin{array}{c}\text { MO } \\
\text { associada }^{(3)}\end{array}$ \\
\hline & \multicolumn{3}{|c|}{------------ $\left(\mathrm{Mg} \mathrm{ha}^{-1}\right.$ de $\left.\mathrm{C}\right)$----------- } \\
\hline & \multicolumn{3}{|c|}{$0-2,5 \mathrm{~cm}$} \\
\hline PC & $7,39 b$ & $1,52 b$ & $6,25 \mathrm{ab}$ \\
\hline PD-Rotação I & $10,76 \mathrm{a}$ & $3,33 \mathrm{ab}$ & $7,43 \mathrm{ab}$ \\
\hline PD-Rotação II & $10,30 \mathrm{a}$ & $3,40 \mathrm{ab}$ & $6,90 \mathrm{ab}$ \\
\hline PD-Rotação III & $10,03 \mathrm{ab}$ & $3,89 \mathrm{a}$ & $6,14 b$ \\
\hline \multirow[t]{2}{*}{ PD Rotação IV } & $11,31 \mathrm{a}$ & $3,62 \mathrm{a}$ & $7,69 \mathrm{a}$ \\
\hline & \multicolumn{3}{|c|}{$2,5-5 \mathrm{~cm}$} \\
\hline $\mathrm{PC}$ & $6,80 \mathrm{~b}$ & $1,53 \mathrm{a}$ & $5,99 \mathrm{a}$ \\
\hline PD-Rotação I & 7,90ab & $1,23 \mathrm{a}$ & $6,67 \mathrm{a}$ \\
\hline IPD-Rotação II & $8,09 \mathrm{a}$ & $1,51 \mathrm{a}$ & $6,58 \mathrm{a}$ \\
\hline PD-Rotação III & $7,56 a b$ & $1,21 \mathrm{a}$ & $6,35 \mathrm{a}$ \\
\hline \multirow[t]{2}{*}{ PD Rotação IV } & $7,86 \mathrm{ab}$ & $1,29 \mathrm{a}$ & $6,57 \mathrm{a}$ \\
\hline & \multicolumn{3}{|c|}{$5-10 \mathrm{~cm}$} \\
\hline $\mathrm{PC}$ & $14,31 \mathrm{a}$ & $2,66 \mathrm{a}$ & $12,74 a$ \\
\hline PD-Rotação I & $13,49 b$ & $1,39 b$ & $12,10 \mathrm{a}$ \\
\hline PD-Rotação II & $13,58 \mathrm{ab}$ & $1,40 \mathrm{~b}$ & $12,18 \mathrm{a}$ \\
\hline PD-Rotação III & $13,21 b$ & $1,32 b$ & $11,89 \mathrm{a}$ \\
\hline \multirow[t]{2}{*}{ PD Rotação IV } & $14,33 \mathrm{ab}$ & $1,40 \mathrm{~b}$ & $12,93 \mathrm{a}$ \\
\hline & \multicolumn{3}{|c|}{$10-15 \mathrm{~cm}$} \\
\hline $\mathrm{PC}$ & $13,53 \mathrm{a}$ & $2,22 \mathrm{a}$ & $12,10 \mathrm{a}$ \\
\hline PD-Rotação I & $12,87 \mathrm{a}$ & $1,15 b$ & $11,72 \mathrm{a}$ \\
\hline PD-Rotação II & $12,71 \mathrm{a}$ & $1,22 b$ & $11,49 a$ \\
\hline PD-Rotação III & $13,19 \mathrm{a}$ & $1,31 b$ & $11,88 \mathrm{a}$ \\
\hline \multirow{2}{*}{ PD-Rotação IV } & $13,10 \mathrm{a}$ & $1,37 \mathrm{~b}$ & $11,73 \mathrm{a}$ \\
\hline & \multicolumn{3}{|c|}{$15-20 \mathrm{~cm}$} \\
\hline PC & $12,27 \mathrm{a}$ & $1,81 \mathrm{a}$ & $11,48 \mathrm{a}$ \\
\hline PD-Rotação I & $11,04 \mathrm{a}$ & $0,80 \mathrm{a}$ & $10,25 b$ \\
\hline PD-Rotação II & $11,81 \mathrm{a}$ & $0,88 \mathrm{a}$ & $10,93 \mathrm{ab}$ \\
\hline PD-Rotação III & $11,88 \mathrm{a}$ & $0,75 a$ & $11,13 \mathrm{ab}$ \\
\hline \multirow[t]{2}{*}{ PD-Rotação IV } & $12,36 \mathrm{a}$ & $1,05 \mathrm{a}$ & $11,31 \mathrm{a}$ \\
\hline & \multicolumn{3}{|c|}{$0-20 \mathrm{~cm}$} \\
\hline $\mathrm{PC}$ & $54,30 \mathrm{~b}$ & $5,74 b$ & $48,56 a$ \\
\hline PD-Rotação I & $56,07 \mathrm{ab}$ & $7,89 \mathrm{a}$ & $48,18 \mathrm{a}$ \\
\hline PD-Rotação II & $56,49 \mathrm{ab}$ & $8,41 \mathrm{a}$ & $48,08 \mathrm{a}$ \\
\hline PD-Rotação III & $55,86 a b$ & $8,48 \mathrm{a}$ & $47,38 \mathrm{a}$ \\
\hline PD-Rotação IV & $58,96 \mathrm{a}$ & $8,73 \mathrm{a}$ & $50,23 \mathrm{a}$ \\
\hline
\end{tabular}

${ }^{(1)}$ Médias seguidas de letras iguais na coluna não diferem entre si pelo teste de Tukey a 5\% de probabilidade. (2)PC: preparo convencional; PD: plantio direto; Rotação I: guandu-anão, milheto/soja, milho; Rotação II: crotalária juncea, sorgo/soja, milho; Rotação III: girassol, aveiapreta/soja, milho; Rotação IV: nabo forrageiro, milho/soja, milho. ${ }^{(3)} \mathrm{MO}$ associada aos minerais $=$ MO total - MO particulada. das superficiais do solo resultam em efeitos importantes em relação à ciclagem de nutrientes, agregação, atividade microbiana, movimento e armazenamento de água, e troca de gases com a atmosfera.

Os estoques de $\mathrm{C}$ na $\mathrm{MO}$ particulada representaram, na camada de $0-20 \mathrm{~cm}$, de $11 \%$ a $15 \%$ do COT, e os maiores valores ocorreram no solo em PD (Tabela 2). No solo em PC a distribuição dos teores de C na MO particulada foi mais uniforme na camada de solo gradeada, resultante da incorporação periódica dos resíduos vegetais (Figura 1).

Houve maior sensibilidade do estoque de $\mathrm{C}$ na $\mathrm{MO}$ particulada às alterações no manejo em comparação ao estoque de $\mathrm{C}$ orgânico total (Figura 2). Em comparação ao solo em PC com culturas estivais, o PD com culturas estivais e outonais promoveu um aumento de $37 \%$ a $52 \%$ no estoque de $\mathrm{C}$ na $\mathrm{MO}$ particulada da camada de 0-20 cm, e foi significativo nos quatro sistemas de cultura (Tabela 2). Por sua vez, o aumento relativo no estoque de COT foi inferior a $10 \%$. Estes resultados demonstram que o estoque de $\mathrm{C}$ na $\mathrm{MO}$ particulada constitui-se indicador mais sensível à qualidade dos sistemas de manejo do solo, possibilitando verificar o efeito a curto prazo (Chan, 1997; Needelman et al., 1999; Bayer et al., 2001, 2002). De forma similar ao COT, os maiores incrementos dos estoques de $\mathrm{C}$ na $\mathrm{MO}$ particulada ocorreram nas camadas superficiais do solo, e corresponderam a aumentos variando entre $192 \%$ e $241 \%$ na camada de $0-2,5 \mathrm{~cm}$, como pode ser calculado a partir dos resultados da Tabela 2.

Em comparação ao solo em PC com culturas estivais, o efeito positivo do PD com culturas estivais e outonais sobre a MO particulada pode estar relacionado à maior adição anual e manutenção dos resíduos vegetais na superfície do solo, em adição à diminuição da atividade microbiana pela redução da temperatura do solo e menor aeração, e principamente, à proteção física da $\mathrm{MO}$ no interior de agregados. A agregação é relacionada à proteção física de frações lábeis da $\mathrm{MO}$ a biodegradação (Balesdent et al., 2000). Em adição aos maiores teores de MO no solo em PD, a menor exposição dos agregados na superfície do solo a repetidos ciclos de umedecimento e secamento, ao impacto das gotas de chuva e à saturação rápida (Bresson \& Boiffin, 1990) contribuem para a maior estabilidade de agregados, no interior dos quais a fração orgânica encontra-se fisicamente protegida dos microrganismos e de suas enzimas (Balesdent et al., 2000). 

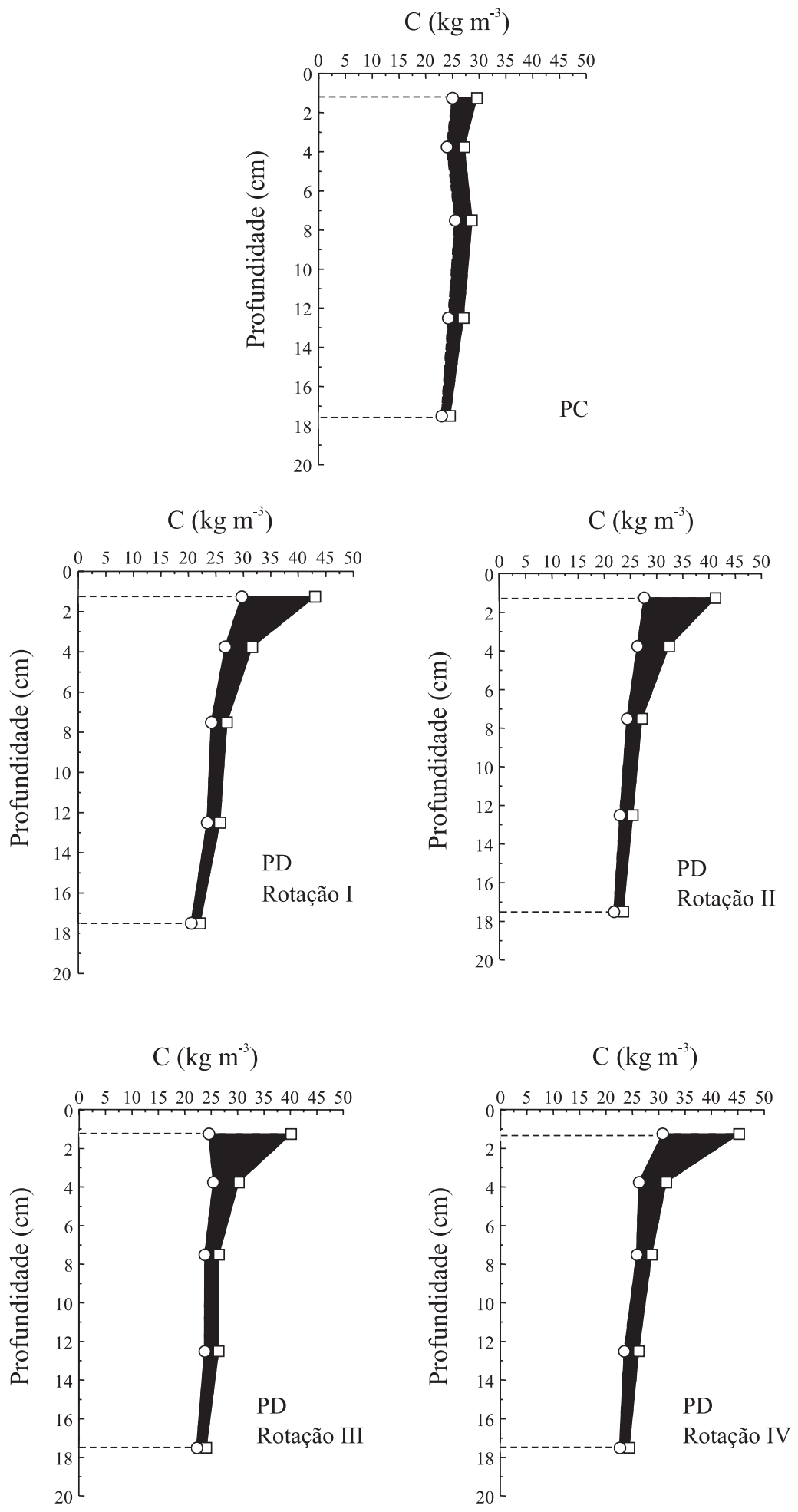

Figura 1. Distribuição dos teores de C na MO particulada ( $\square$ ) e MO associada aos minerais (O) de um Latossolo Vermelho ácrico sob preparo convencional (PC) e plantio direto (PD). Rotação I: guandu-anão, milheto/soja, milho; Rotação II: crotalária juncea, sorgo/soja, milho; Rotação III: girassol, aveiapreta/soja, milho; Rotação IV: nabo forrageiro, milho/soja, milho. 
$\mathrm{O}$ estoque de $\mathrm{C}$ na $\mathrm{MO}$ associada aos minerais não foi afetado pelos sistemas de manejo nas diferentes camadas de solo, o que refletiu-se em estoques de $\mathrm{C}$ nesta fração estatisticamente iguais na camada de $0-20 \mathrm{~cm}$ (Tabela 2). O pequeno período de condução do sistema PD (seis anos) facilita o entendimento do não-efeito dos sistemas de manejo na MO associada aos minerais, a qual apresenta um avançado estágio de humificação e é altamente estável devido a sua interação com a fração mineral (estabilidade química) e localização no interior de microagregados ( $<53 \mu \mathrm{m})$ estáveis (proteção física), além da sua maior recalcitrância química decorrente da sua composição (Bayer, 1996). Como a MO associada aos minerais apresenta uma ciclagem bem mais lenta, no que se refere à sua formação e decomposição, é necessário um período maior para que a alteração dos sistemas de manejo tenha efeito no estoque de $\mathrm{C}$ desta fração.

Outro aspecto importante é que o efeito do manejo na MO protegida em microagregados deve ser dependente da textura e, principalmente, da mineralogia do solo, as quais influenciam a estabilidade destes microagregados. Nesse sentido, em adição ao pequeno período trasncorrido, a não-ocorrência de variação da

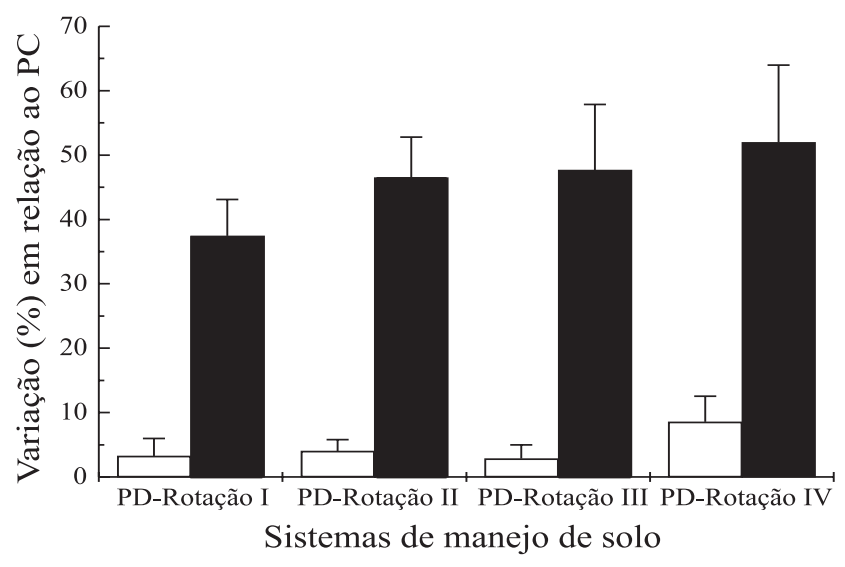

Figura 2. Variação porcentual dos estoques de C orgânico total ( $\square$ ) e na MO particulada ( $\square$ ) na camada de 0-20 cm de um Latossolo Vermelho ácrico sob plantio direto (PD) com diferentes sistemas de rotação de culturas (Rotação I: guanduanão, milheto/soja, milho; Rotação II: crotalária juncea, sorgo/ soja, milho; Rotação III: girassol, aveia-preta/soja, milho; Rotação IV: nabo forrageiro, milho/soja, milho), em relação ao solo em preparo convencional (PC). Barra em cada coluna representa desvio-padrão da média.
MO associada aos minerais provavelmente seja decorrente do pequeno efeito dos sistemas de manejo na ruptura e formação de microagregados $<53 \mu \mathrm{m}$ neste Latossolo oxídico, cujo principal agente cimentante são os óxidos de ferro pouco cristalinos (Pinheiro-Dick \& Schwertmann, 1996; Muggler et al., 1999). A proteção da MO em microagregados altamente estáveis, os quais praticamente não são afetados pelo manejo, é coerente com a pequena diferença que tem sido verificada nos estoques de MO entre o PD e o PC em solos de textura argilosa e com altos teores de óxidos de ferro no Sul do Brasil (Bayer, 1996; Freixo et al., 2002).

Quanto à retenção de dióxido de carbono atmosférico, o acúmulo de $\mathrm{C}$ na fração lábil da $\mathrm{MO}$ neste Latossolo Vermelho implica que o PD e o aporte de resíduos devam ser mantidos continuamente, pois a sua interrupção pode resultar num efluxo líquido de $\mathrm{C}$ para a atmosfera. Também é desconhecido o efeito que o aumento antrópico da temperatura previsto para as próximas décadas poderá apresentar sobre o $\mathrm{C}$ armazenado em formas lábeis da MO do solo.

\section{Conclusões}

1. O plantio direto aumenta os estoques de carbono orgânico do solo em comparação ao preparo convencional, sendo este efeito restrito às camadas superficiais e dependente do sistema de cultura.

2. O acúmulo de carbono no solo em plantio direto ocorre preferencialmente na matéria orgânica particulada, a qual é mais sensível do que o carbono orgânico total às alterações no manejo do solo.

3. Em períodos curtos (seis anos), o plantio direto não tem efeito no estoque de carbono na matéria orgânica associada aos minerais em Latossolos argilosos oxídicos.

\section{Referências}

Associação de Plantio Direto no Cerrado (Brasília, DF). Plantio direto tropical. Disponível em: <http://www.apdc.com.br〉. Acesso em: 25 mar. 2004.

BALESDENT, J.; CHENU, C.; BALABANE, M. Relationship of soil organic matter dynamics to physical protection and tillage. Soil and Tillage Research, v.53, p.215-230, 2000.

BAYER, C. Dinâmica da matéria orgânica em sistemas de manejo de solos. 1996. 241p. Tese (Doutorado) - Universidade Federal do Rio Grande do Sul, Porto Alegre.

BAYER, C.; MARTIN-NETO, L.; MIELNICZUK, J.; SANGOI, L. Changes in soil organic matter fractions under subtropical no-till 
cropping systems. Soil Science Society of America Journal, v.65, p.1473-1478, 2001.

BAYER, C.; MIELNICZUK, J. Dinâmica e função da matéria orgânica. In: SANTOS, G.A.; CAMARGO, F.A.O. (Ed.). Matéria orgânica do solo: fundamentos e caracterização. Porto Alegre: Gênesis, 1999. p.9-26.

BAYER, C.; MIELNICZUK, J.; MARTIN-NETO, L.; ERNANI, P.R. Stocks and humification degree of organic matter fractions as affected by no-tillage on a subtropical soil. Plant and Soil, v.238, p.133-140, 2002.

BRESSON, L.M.; BOIFFIN, J. Morphological characterization of soil crust development stages on an experimental field. Geoderma, v.47, p.301-325, 1990.

BUYANOVSKY, G.A.; ASLAM, M.; WAGNER, G.H. Carbon turnover in soil physical fractions. Soil Science Society of America Journal, v.58, p.1167-1173, 1994.

CAMBARDELLA, C.A.; ELLIOT, E.T. Particulate soil organicmatter changes across a grassland cultivation sequence. Soil Science Society of America Journal, v.56, p.777-783, 1992.

CASSOL, E.A. Erosão do solo: influência do uso agrícola, do manejo e preparo do solo. Porto Alegre: Secretaria da Agricultura, Instituto de Pesquisa em Recursos Naturais Não Renováveis, 1984. 40p. (Boletim Técnico, 15).

CHAN, K.Y. Consequences of changes in particulate organic carbon in vertisols under pasture and cropping. Soil Science Society of America Journal, v.61, p.1376-1382, 1997.

CORAZZA, E.J.; SILVA, J.E.; RESCK, D.V.S.; GOMES, A.C. Comportamento de diferentes sistemas de manejo como fonte ou depósito de carbono em relação à vegetação de cerrado. Revista Brasileira de Ciência do Solo, v.23, p.425-432, 1999.

FELLER, C.; BEARE, M.H. Physical control of soil organic matter dynamics in the tropics. Geoderma, v.79, p.69-116, 1997.

FRANZLUEBBERS, A.J.; ARSHAD, M.A. Particulate organic carbon content and potential mineralization as affected by tillage and texture. Soil Science Society of America Journal, v.61, p.1382-1386, 1997.

FREIXO, A.F.; MACHADO, P.L.O.A.; SANTOS, H.P.; SILVA, C.A.; FADIGAS, F.S. Soil organic and fractions of Rhodic Ferralsol under the influence of tillage and crop rotation systems in southern Brazil. Soil and Tillage Research, v.64, p.221-230, 2002.

GOLCHIN, A.; OADES, J.M.; SKJEMSTAD, J.O.; CLARKE, P. Study of free and occluded particulate organic matter in soils by solid state ${ }^{13} \mathrm{C} \mathrm{CP} / \mathrm{MAS}$ NMR spectroscopy and scanning electron microscopy. Australian Journal of Soil Research, v.32, p.285309, 1994.

MENDONÇA, E.S. Oxidação da matéria orgânica e sua relação com diferentes formas de alumínio de Latossolos. Revista Brasileira de Ciência do Solo, v.19, p.25-30, 1995.

MUGGLER, C.C.; GRIETHUYSEN, C. van; BUURMAN, P.; PAPE, T. Aggregation, organic matter, and iron oxide morphology in oxisols from Minas Gerais, Brazil. Soil Science, v.164, p.759-770, 1999.

NEEDELMAN, B.A.; WANDER, M.M.; BOLLERO, G.A.; BOAST, C.W.; SIMS, G.K.; BULLOCK, D.G. Interaction of tillage and soil texture: biologically active soil organic matter in Illinois. Soil Science Society of America Journal, v.63, p.1326-1334, 1999.

NELSON, P.W.; SOMMERS, C.E. Total carbon, organic carbon and organic matter. In: PAGE, A.L. (Ed.). Methods of soil analysis. Madison: Soil Science Society of America, 1982. pt.2, p.539-579 (SSSA. Agronomy Monograph, 9).

PINHEIRO-DICK, D.; SCHWERTMANN, U. Microaggregates from Oxisols and Inceptisols: dispersion through seletive dissolutions and physico-chemical treatments. Geoderma, v.74, p.49-63, 1996.

REICOSKY, D.C.; KEMPER, W.D.; LANGDALE, G.W.; DOUGLAS, C.L.; RASMUNSSEN, P.E. Soil organic matter changes resulting from tillage and biomass production. Journal of Soil and Water Conservation, v.50, p.253-261, 1995.

RESCK, D.V.S. O plantio direto como alternativa de sistema de manejo e conservação do solo e da água na região dos cerrados. In: CONGRESSO BRASILEIRO DE CIÊNCIA DO SOLO, 30., 1997, Rio de Janeiro. Anais. Rio de Janeiro: Sociedade Brasileira de Ciência do Solo, 1997. 21p. Palestras, CD-ROM.

SILVA, J.E.; LEMAINSKI, J.; RESCK, D.V.S. Perdas de matéria orgânica e suas relações com a capacidade de troca catiônica em solos da região de cerrados do oeste baiano. Revista Brasileira de Ciência do Solo, v.18, p.541-547, 1994.

SIX, J.; ELLIOT, E.T.; PAUSTIAN, K.; DORAN, J.W. Aggregation and soil organic matter accumulation in cultivated and native grassland soils. Soil Science Socienty of America Journal, v.62, p.13671377, 1998.

WANDER, M.M.; BIDART, M.G.; AREF, S. Tillage impacts on depth distribution of total and particulate organic matter in three Illinois soils. Soil Science Society of America Journal, v.62, p.1704-1711, 1998.

$\overline{\text { Recebido em } 28 \text { de agosto de } 2003 \text { e aprovado em } 13 \text { de abril de } 2004}$ 